\title{
Do we practice what we preach? A qualitative assessment of resident-preceptor interactions for adherence to evidence-based practice
}

\author{
Jon C. Tilburt MD MPH, ${ }^{1}$ Rajesh S. Mangrulkar MD, ${ }^{2}$ Susan Dorr Goold MD MA MHSA MA, ${ }^{3}$ \\ Nazema Y. Siddiqui $\mathrm{MD}^{4}$ and Joseph A. Carrese MD MPH \\ ${ }^{1}$ Assistant Professor, Division of General Internal Medicine and the Program in Professionalism and Bioethics, Mayo Clinic, Rochester, MN, USA \\ ${ }^{2}$ Clinical Assistant Professor, ${ }^{3}$ Associate Professor, Division of General Internal Medicine, University of Michigan Medical Center, Ann Arbor, MI, \\ USA \\ ${ }^{4}$ Fellow, Department of Obsetrics and Gynecology, Duke University Medical Center, Durham, NC, USA \\ ${ }^{5}$ Associate Professor, Division of General Internal Medicine, Johns Hopkins Bayview Medical Center, and the Johns Hopkins Berman Institute of \\ Bioethics, Johns Hopkins University, Baltimore, MD, USA
}

\author{
Keywords \\ evidence-based medicine, graduate medical \\ education, precepting, qualitative research \\ Correspondence \\ Jon C. Tilburt \\ Division of General Internal Medicine \\ The Program in Professionalism and \\ Bioethics \\ Mayo Clinic \\ Rochester, MN 55905 \\ USA \\ E-mail: tilburt.jon@mayo.edu \\ Accepted for publication: 12 December \\ 2007
}

doi:10.1111/j.1365-2753.2008.00966.x

\begin{abstract}
Background Evidence-based medicine (EBM) is important in training doctors for highquality care. Yet little is known about whether ambulatory precepting incorporates the concepts and principles of EBM.

Methods The authors observed and audiotaped 95 internal medicine residency precepting interactions and rated interactions using a qualitative analytic template consisting of three criteria: (1) presence of clinical questions; (2) presence of an evidence-based process; and (3) resident ability to articulate a clinical question.

Results Sixty-seven of 95 audio tapes (71\%) were of acceptable quality to allow template analysis. Thirty (45\%) contained explicit clinical questions; 11 (16\%) included an evidence-based process. Resident ability to articulate a clinical question when prompted was rated as at least 'fair' in 59 of 67 interactions (88\%).

Conclusions EBM was not optimally implemented in these clinics. Future research could explore more systematically what factors facilitate or impair the use of EBM in the real-time ambulatory training context.
\end{abstract}

\section{Background}

Evidence-based medicine (EBM) involves an explicit process: asking clinical questions, acquiring information from reliable resources, appraising that information, applying the information and assessing the success of the process [1,2,3]. Teaching the process of EBM should be an important objective of residency training so that it is eventually integrated into patient care [4]. The ambulatory training setting provides opportunities for EBM teaching, where faculty are present and resources are accessible. However, there is little data about the extent to which important elements of this process are present in resident-preceptor interactions in the ambulatory setting.

EBM teaching interventions have proven efficacious in structured educational exercises [5,6,7], but attempts to observe adherence to evidence-based practice in real-time behaviour have been limited [8]. Qualitative methods may provide a valid baseline description of doctor behaviours most amenable to EBM interven- tions $[9,10,11]$. Yet, despite attempts by a few investigators to describe actual teaching contexts $[8,12]$, we are not aware of any studies that have successfully used both observation and verbatim audiotaping methods to assess EBM behaviour in real-time resident-preceptor interactions in internal medicine outpatient clinics.

The objective of this study was to assess the content and quality of resident-faculty preceptor interactions using three basic criteria of evidence-based practice in internal medicine ambulatory continuity clinics.

\section{Methods}

\section{Setting}

We observed resident-preceptor interactions in clinic mentor rooms at four sites affiliated with a university-based internal medicine residency programme in the Midwestern United States in 
October 2001. Mentor rooms contained print and computer-based resources where one faculty doctor serves as preceptor for two or three residents. We defined a precepting interaction as the entirety of the resident's experience in the mentor room for one patient case. In this programme preceptors must discuss in detail all patient visits reviewing the chief compliant, history of present illness, past medical history, allergies, social and family history, examination, data, as well as an assessment and plan at the time of the patient visit. In addition, for new patient visits, they are required to examine the patient. Residents are given 30 minutes to complete a return patient visit and 1 hour to complete a new patient visit. In this time period, they are required to discuss the case with their supervising faculty doctor and seek out the information needed to make a sound clinical decision.

\section{Study population}

We analysed a data set of audio transcripts collected from a preexisting study [13]. The residency programme we studied trains doctors in the inpatient setting at a university-based, tertiary referral hospital, and partners with affiliated, university-run ambulatory satellite facilities for the ambulatory component of residency training. Residents in the programme learn the principles of EBM in a sequential EBM seminar series delivered longitudinally over the residents' 3-year training, and in the scheduled educational events of the residency programme (informally during morning reports, teaching rounds, etc.). The EBM curriculum was a major component of the month-long ambulatory block curriculum which residents participated in three or four times over the course of their 3 -year residency. During these months, they participated in daily presentations of evidence-based reviews, and weekly 2-hour sessions on the principles of EBM consisting of a minimum of 24-32 hours of instruction time over the course of the 3-year residency. This study was approved by the sponsoring Institutional Review Board. Written informed consent was obtained from all participating residents and faculty.

\section{Data collection and management}

The observing investigator (NS) used a semi-structured data collection form to record field notes of observations developed in collaboration with three investigators (JT, SDG, RM). The form was field tested by two investigators (JT, NS), who observed two half-day clinic sessions to assess and subsequently modify the data collection form. The observer (NS) watched residents in half-day sessions, recorded field notes of her observations and audiotaped conversations. The observer was a recent medical graduate with training in medical informatics. She was not well known to study participants but was familiar with the way these clinics worked based on her prior experience as a student. She followed a training protocol developed by the lead investigators (JT, RM) to focus on 'information exchange behaviour'. She was trained before the observation period by doing parallel observation of clinic events with one lead investigator. Field notes captured nonverbal information exchange behaviours not recorded on audio tape and provided confirmatory information for transcript analysis. After the precepting interaction, the observer asked each resident, 'What was your most important question for this patient?' and recorded their answers. We combined these answers with field notes and transcripts of audio recordings to create a single transcript document for each precepting interaction.

Tapes were transcribed by a professional medical transcription company and then reviewed by the lead investigator to insure accuracy of content. Tapes deemed undecipherable by the transcriptionists were reviewed separately by a second medical transcriptionist and if possible were re-transcribed with the assistance of the lead investigator (JT) to insure accuracy. All audio tapes were reviewed by at least one investigator before and after transcription to insure accuracy of transcript content.

\section{Template development and analysis}

Unlike our previous study in which we describe verbal and nonverbal information seeking behaviours in the social context of precepting [13], this analysis evaluated transcripts for existing elements of evidence-based medicine as they apply to the precepting context using an analytic template. In qualitative research, a template refers to a set of a priori constructs through which to view and analyse observed behaviour [14]. We devised a semistructured instrument to apply the template comprised of three general criteria of evidence-based precepting: (1) presence of an explicit, clinical question that was raised spontaneously in the precepting interaction; (2) presence of an explicit evidence-based process; and (3) the ability of the resident to articulate a clinical question when prompted (Appendix). We selected these criteria based on the literature [15] and on feedback from two academic general internal medicine faculty groups (12 faculty total) not affiliated with the study site.

Explicit clinical questions could be phrased either as an interrogative statement ('What is the diagnostic approach for pharyngitis?') or as a declarative statement ('I need to know more about beta-blockers.') so long as it was a clear expression of clinical uncertainty. A well-built clinical question is the initial key step in achieving evidence-based practice [16]. Because this was a realtime assessment, we did not require that a question contain all the elements of a well-built question as outlined by Richardson et al. [16]. Nevertheless, our review of the extensive literature on the importance clinical questions in the EBM process prompted our inclusion of them as a key criterion for our analytic template.

To be fair in assessing the first two criteria, we thought it was important to treat the resident-faculty dyad together. Presence of an evidence-based process was determined by whether or not there was evidence of behaviour intended to seek out, use or reference research evidence or another published source of information during the precepting interaction. Seeking out another person to answer a question, for instance, was not considered an evidencebased process unless in the process of engaging that person research evidence or another published source of information was accessed or referred to in some way.

We defined the degree to which the resident was able to articulate a focused, detailed and specific clinical question, when invited in the following way. 'Fair' or better responses, contained at least one element of specificity regarding the patient, their condition or the proposed intervention. 'Poor'-quality questions were defined as having no specificity regarding the patient, the proposed intervention, the comparison or the outcome of interest. Because this was a real-time evaluation we elected not to use strict criteria for question quality, such as the four-part PICO (population/patient, 
Table 1 Three criteria of evidence-based practice used to rate precepting interactions

\begin{tabular}{ll}
\hline Criteria & Definition \\
\hline $\begin{array}{l}\text { 1. Sponteous clinical question (Y/N) } \\
\text { 2. EBM process (Y/N) }\end{array}$ & Any expression of clinical uncertainty that arouse spontaneously in the precepting interaction. \\
& Any effort to incorporate the subsequent steps of the EBM process: Acquire, Appraise, Apply and/or \\
& Assess. \\
& Acquire: Searching the appropriate electronic or print databases for information. \\
& Appraise: Determining which evidence is the best of the evidence found and whether it is of sufficient \\
quality to be useable. & \\
Applying: Using the evidence in the relevant clinical circumstance. & Assess: Determining whether one's application of the evidence went well and was appropriate to the \\
& circumstance. \\
& The degree to which the resident was able to articulate a focused, detailed and specific clinical question, \\
3. Ability to articulate a question & when invited. 'Fair' or better quality responses contained at least one element of specificity regarding \\
(poor, fair, good, excellent) & the patient, their condition or the proposed intervention.
\end{tabular}

EBM, evidence-based medicine.

intervention, comparison and outcome) criteria [16]. Rather we were looking for practical indications that residents were able to formulate with at least some specificity a question that may be sufficient to stimulate other evidence-based behaviours.

For criteria 1 and 2, we rated transcripts using dichotomous scoring categories (Yes/No). For criterion 3 we rated transcripts on a 1-4 Likert scale $(1=$ poor, 2 = fair, $3=\operatorname{good}, 4=$ excellent $)$ and subsequently dichotomized that scale, as we were primarily interested in identifying whether residents could articulate at least a fair quality question.

We sought to ensure the trustworthiness of our interpretation process in a manner consistent with accepted methods of qualitative research [14]. First, using the criteria we had established by reviewing the literature and consulting with experts, two investigators (JT, JC) independently analysed a random subset of 20 transcript documents (30\% of total) using the three criteria ( $\kappa=0.63,0.56,0.64$, respectively, for each of the three criteria) (Table 1). The two investigators then met to resolve scoring differences with consensus ratings for those 20 transcripts and agreed on the meaning of the constructs going forward. One investigator (JT) then analysed all remaining unanalysed transcript documents (47) using the analysis instrument and the identified criteria. We found we could not interpret interactions accurately based on field notes alone because of the subtle nature of precepting interactions. Therefore, we scored only those transcripts in which we had good quality transcript and field note data.

\section{Results}

Of the 147 residents in the programme, we observed 70 of 89 eligible for inclusion during the study period and 28 of 34 eligible precepting faculty in 95 interactions over 17 half-day clinic sessions. Data collection occurred during October 2001. Transcripts from 28 of these interactions were eliminated from this analysis because of poor audio transcript quality. Participating residents and faculty were similar to their institutional colleagues (Table 2). Faculty members were well-trained, mid-career academic internists. At least half were fellowship trained or had completed a year as chief resident. Fifty percent of faculty reported participating in faculty development courses to improve clinical teaching and $50 \%$ reported completing some sort of EBM training.

\section{Criterion 1: explicit, spontaneous clinical questions}

We found evidence of spontaneous clinical questions in 30 of 67 interactions $(45 \%)$. For example, while discussing a patient with pharyngitis, a resident asked, 'How do you usually manage this, where you think it's viral, but you've done a culture?' In another example, an attending asked 'Which is a better thing for her, from what we know of the medical literature: to start the triamterene/ HCTZ back or to give her an ACE inhibitor instead?' In these questions, residents and attendings demonstrated willingness to seek information, express uncertainty and think critically. However, when a clinical question was not raised (37 of 67 interactions), the resident and attending were nonetheless able to get the work of patient care accomplished through mutual dialogue.

\section{Criterion 2: explicit EBM process}

An EBM process was present in 11 of 67 (16\%) interactions. For instance, a resident, unsure about whether to prescribe antibiotics for a patient with sinus symptoms, presented the case to the attending, who initially wanted to treat the patient with antibiotics. In the field notes that follow, they acknowledge clinical uncertainty and decide to seek out a clinical practice guideline.

Resident . . . looks at clinical guidelines [an institutional updated version available at http://cme.med.umich.edu/pdf/ guideline/rhino05.pdf] - looks up sinusitis ... reads off ... says if a patient has one indicator, then $20 \%$ chance of having sinusitis; if two, then $40 \%$ chance of having sinusitis.

Attending relents ... and says to aggressively treat with decongestants.

They conclude by recommending decongestants and decide to reserve antibiotics for refractory symptoms, demonstrating an explicit attempt to acquire and apply evidence.

\section{Criterion 3: resident ability to formulate a clinical question}

We rated the residents' ability to formulate a clinical question, when prompted ('What was your most important question for this patient?') as 'fair' or better in 59 of 67 (88\%) precepting interac- 
Table 2 Characteristics of 70 participating residents and 21 faculty who were observed in 95 precepting interactions in relation to their respective populations

\begin{tabular}{|c|c|c|}
\hline \multirow[b]{2}{*}{ Characteristics } & \multicolumn{2}{|c|}{ Resident characteristics* } \\
\hline & $\begin{array}{l}\text { Residency } \\
\text { population } \\
(n=147)\end{array}$ & $\begin{array}{l}\text { Participating } \\
\text { residents } \\
(n=70)\end{array}$ \\
\hline Men (\%) & $102(69)$ & $48(69)$ \\
\hline PGY 1 (\%) & $53(36)$ & $28(40)$ \\
\hline PGY 2 (\%) & $44(30)$ & $19(27)$ \\
\hline PGY 3 (\%) & $44(30)$ & $21(30)$ \\
\hline \multirow[t]{3}{*}{ PGY 4 (\%) } & $6(4)$ & $2(3)$ \\
\hline & \multicolumn{2}{|c|}{ Faculty Characteristics* ${ }^{*}$} \\
\hline & $\begin{array}{l}\text { Faculty } \\
\text { population } \\
(n=29)\end{array}$ & $\begin{array}{l}\text { Participating } \\
\text { faculty } \\
(n=21)\end{array}$ \\
\hline Mean age in years (SD) & $40(6.1)$ & $41(6.2)$ \\
\hline Men (\%) & $19(66)$ & $14(66)$ \\
\hline Mean years of precepting (SD) & $9.0(5.1)$ & $10(5.1)$ \\
\hline Years out of medical school (SD) & $13(5.3)$ & $14(5.3)$ \\
\hline Completed fellowship or chief residency (\%) & $17(50)$ & $13(57)$ \\
\hline Completed faculty development (\%) & $17(59)$ & $11(50)$ \\
\hline Completed EBM training (\%) & $14(48)$ & $10(50)$ \\
\hline
\end{tabular}

* $P$-values compare groups with population as a whole using test comparing equality of means or proportions. P-values for all comparisons were not statistically significant $(>0.05)$.

${ }^{\dagger} A$ total of 28 of 34 eligible faculty participated in the study. Twenty-nine of 34 responded to a survey to ascertain participant characteristics. Twenty-three of the 28 observed faculty were included in the analysis for this report (others excluded because of transcript quality). Of those 23 , we were able to gather characteristics from 21 of them.

EBM, evidence-based medicine; SD, standard deviation. tions. A fair quality response to our prompting as we defined it contained at least one element of specificity regarding the patient, their condition or the proposed intervention. Resident responses ranged in their degree of detail. The following response represents one of the better quality responses to our inquiry.

How to evaluate potential liver function test abnormalities in patients on 'statins'?

In this response, the resident states a particular test as well as relevant features of the patient's condition. Other responses had less specificity, but were nevertheless relevant to the case, for instance,

How much pain is the patient still having?

In this response, we hear what the problem is, but little is said about specific features of the problem at hand. We rated such questions as 'fair'. Occasionally (8 of 67 transcripts, 12\%), when prompted, residents were not able to articulate a clinical question of even fair quality. In one such example, the resident had just completed a conversation with an attending about the diagnostic approach to inguinal lymphadenopathy in the setting of a patient with a history of ovarian cysts. His response to our query ('What was your most important question for this patient?') was, 'What to do with this patient?' We rated this response on as 'poor' because it contained virtually no specific information that could shape the direction of decision making. Another time a resident said 'None' in response to the observer's inquiry. This followed a discussion of a thyroid biopsy. But on the whole residents did seem to be able to articulate a clinical question of fair or higher quality in the vast majority of cases.

\section{Discussion}

In this study, we observed resident-preceptor interactions for the presence of three basic criteria of EBM using a qualitative analytic approach. Formulating an explicit clinical question is the initial fundamental step in the EBM process (criteria 1) [16]. We found this step being exercised in a minority of resident-preceptor interactions. Nevertheless, clinical questions were still nearly three times as common in our observations as all other steps in the EBM process (criteria 2). We observed these findings in an academic programme with talented residents and faculty who are highly trained and who dedicate time specifically to precepting. When prompted, residents were able to articulate a clinical question (criteria 3) with some degree of specificity in the vast majority of cases. Residents are clearly able to formulate questions in most situations and yet do so less frequently. Therefore, future research may benefit from further defining barriers to and facilitators of succinct clinical question formulation in these interactions.

This study had some limitations. We did not observe the same resident-faculty pairs over a whole clinic session. Faculty may make trade-offs in their approach with residents over the course of half-day clinic sessions. Our approach may not have adequately accounted for those pragmatic considerations. We chose to sample a broader range of more isolated interactions, believing that the diverse personalities and styles of residents and faculty would enrich our analysis. Furthermore, EBM is only one dimension of exemplary precepting; in order to assess precepting quality more 
globally, one could account for a range of behaviours beyond EBM practice using existing models of learning theory for faculty and residents $[17,18]$.

\section{Conclusions}

This qualitative, observational approach allowed us to assess the extent of EBM behaviours in real-time resident-preceptor interactions in internal medicine clinics. The study findings add to the growing body of knowledge regarding the applicability of EBM in ambulatory care, particularly with respect to internal medicine training. Future research could explore more systematically the factors that facilitate or impair the use of EBM in the ambulatory training context.

\section{Acknowledgements}

The Division of General Internal Medicine, University of Michigan Health System, provided funding for the study to Dr Mangrulkar. The funder had no role in study design, collection, analysis, interpretation of the data, drafting of the manuscript or in the decision to submit the manuscript.

\section{References}

1. Oxman, A. D., Sackett, D. L. \& Guyatt, G. H. (1993) Users' guides to the medical literature. I. How to get started. The Evidence-Based Medicine Working Group. Journal of the American Medical Association, 270, 2093-2095.

2. Sackett, D. L., Strauss, S. E., Richardson, W. S., Rosenberg, W. \& Haynes, R. B. (2000) Evidence-Based Medicine: How to Practice and Teach EBM, 2nd edn. Philadelphia, PA: Churchill Livingston.

3. Sackett, D. L., Rosenberg, W. M. C., Gray, J. A. M., Haynes, R. B. \& Richardson, W. S. (1996) Evidence based medicine: what it is and what it isn't. British Medical Journal, 312 (7023), 71-72.

4. Korenstein, D., Dunn, A. \& McGinn, T. (2002) Mixing it up: integrating evidence-based medicine and patient care. Academic Medicine, 77, 741-742.

5. Cabell, C. H., Schardt, C., Sanders, L., Corey, G. R. \& Keitz, S. A. (2001) Resident utilization of information technology. Journal of General International Medicine, 16, 838-844.

6. Fliegel, J. E., Frohna, J. G. \& Mangrulkar, R. S. (2002) A computerbased OSCE station to measure competence in evidence-based medicine skills in medical students. Academic Medicine, 77, 11571158.

7. Ramos, K. D., Schafer, S. \& Tracz, S. M. (2003) Validation of the Fresno test of competence in evidence based medicine. British Medical Journal, 326, 319-321.

8. Ramos, K. D., Linsheid, R. \& Schafer, S. (2003) Real-time information-seeking behavior of residency physicians. Family Medicine, 35, 257-260.
9. Glenn, J. K., Reid, J. C., Mahaffy, J. \& Shurtlett, H. (1984) Teaching behaviors in the attending-resident interaction. Journal of Family Practice, 18, 297-304.

10. Montori, V. M., Tabini, C. C. \& Ebbert, J. O. (2002) A qualitative assessment of 1st-year internal medicine residents' perceptions of evidence-based clinical decision making. Teaching and Learning in Medicine, 14, 114-118.

11. Greenhalgh, T., Toon, P., Russell, J., Wong, G., Plumb, L. \& MacFarlane, F. (2004) Transferability of principles of evidence-based medicine to improved educational quality: systematic review and case study of an online course in primary health care. British Medical Journal, 326, 142-145.

12. Forsythe, D. E., Buchanan, B. G., Osheroff, J. A. \& Miller, R. A. (1992) Expanding the concept of medical information: an observational study of physician's information needs. Computers in Biomedical Research, 25, 181-200.

13. Tilburt, J. C., Goold, S. D., Siddiqui, N. \& Mangrulkar, R. S. (2007) How do physicians use information in real-time: a qualitative study of internal medicine resident precepting. Journal of Evaluation in Clinical Practice, 13, 772-780.

14. Miller, W. L. \& Crabtree, B. F. (eds) (1999) Doing Qualitative Research, 2nd edn. New York: Sage Publications.

15. Haynes, R. B., Devereaux, P. J. \& Guyatt, G. H. (2002) Clinical expertise in the era of evidence-based medicine and patient choice. ACP Journal Club, 136, A11-A14.

16. Richardson, W. S., Wilson, M. C., Nishikawa, J. \& Hayward, R. S. (1995) The well-built clinical question: a key to evidence-based decisions. ACP Journal Club, 123, A12-A13.

17. Kern, D. E., Branch, W. T. Jr, Jackson, J. L., Brady, D. W., Feldman, M. D., Levinson, W. \& Lipkin, M. Jr. (2005) Teaching the Psychosocial Aspects of Care in the Clinical Setting: Practical Recommendations. Academic Medicine, 80, 8-20.

18. Committee on the Development in the Science of Learning, Committee on Learning Research and Education Practice, Commission on Behavioral Social Sciences Education, National Research Council (2000) How People Learn: Brain, Mind, Experience, and School. Washington, DC: National Academy Press.

\section{Appendix}

\section{Analysis template}

File no.:

Reviewer:

EBM process quality/applicability

1. Was an explicit clinical question articulated in any of the dialogue for the key issue? $(\mathrm{y} / \mathrm{n})$

2. Was EBM process followed? $(\mathrm{y} / \mathrm{n})$

3. Ability of resident to articulate a clinical question when prompted: 1234

( 1 = poor, 2 = fair, $3=$ good, $4=$ excellent $)$ 